\begin{tabular}{|c|l|}
\hline Title & Laser picosecond acoustics with oblique probe light incidence \\
\hline Author(s) & Matsuda, O.; Wright, O. B. \\
\hline Citation & $\begin{array}{l}\text { Review of Scientific Instruments, 74(1), 895-897 } \\
\text { https://doi.org/10.1063/1.1519679 }\end{array}$ \\
\hline Issue Date & 2003 \\
\hline Doc URL & http://hdl.handle.net/2115/14638 \\
\hline Rights & Copyright $\odot 2003$ A merican Institute of Physics \\
\hline Type & article \\
\hline File Information & RSI741.pdf \\
\hline
\end{tabular}

Instructions for use 


\title{
Laser picosecond acoustics with oblique probe light incidence
}

\author{
O. Matsuda ${ }^{\text {a) }}$ and O. B. Wright \\ Department of Applied Physics, Faculty of Engineering, Hokkaido University, Sapporo 060-8628, Japan
}

(Presented on 27 June 2002)

\begin{abstract}
Laser picosecond acoustics involves the excitation and detection of picosecond acoustic strain pulses in thin films with ultrashort light pulses. The use of oblique probe light incidence permits a greater degree of freedom in choosing the optical probing conditions and thereby should allow the extraction of more information about the profile of the propagating acoustic strain pulses. Here, we present a theory for the modulation of light reflected at oblique incidence from a solid containing an acoustic strain distribution. The theory can account for the real and imaginary parts of the reflectance variation, and involves both the effect of the transient surface motion and of the photoelastically modulated dielectric constants in the solid. We show, in particular, how the theory can be applied to extract the contribution to the reflectance change arising from the surface motion in an opaque isotropic solid, thereby allowing direct access to the shape of the acoustic strain pulse propagating therein. () 2003 American Institute of Physics. [DOI: 10.1063/1.1519679]
\end{abstract}

\section{INTRODUCTION}

The technique of laser picosecond acoustics can be used to evaluate the optical, mechanical, and elastic properties of thin films. ${ }^{1,2}$ Ultrashort light pulses are used to generate picosecond acoustic phonon pulses in the sample. These phonon pulses propagate in the sample and are detected by ultrashort probe light pulses through the modulation in reflectance or transmittance caused by the photoelastic effect $^{1}$ or by surface and interface displacements in the sample., ${ }^{2,3}$

Interferometric detection techniques are often used to detect both the real and imaginary parts of the reflectance modulation; the probe light pulses are typically incident on the sample surface at normal or near-normal incidence ${ }^{3,4}$ because rigorous theoretical methods of analysis for single or multilayer films are only available in this case. ${ }^{5,6}$ For standard reflectance and transmittance detection techniques oblique incidence probing is often used mainly because of the convenience in the experimental setups. However, the results have not been analyzed with a complete theory for probing at oblique incidence. This is unfortunate because the use of an oblique probe incidence geometry with proper analysis is expected to increase the information that can be obtained about the sample owing to the extra degrees of freedom introduced by the variable angle of incidence and probe light polarization. Particular advantages of the use of oblique incidence compared to the use of normal incidence for the probe beam are as follows: (1) shear strain pulses can be detected; ${ }^{7,8}$ (2) anisotropic materials can be probed effectively; and (3) signals arising from the photoelastic effect and from surface displacements can be separated. The temporal variation of the surface displacement directly reveals the generated phonon pulse shape. ${ }^{2}$ This is especially important for the analysis of carrier diffusion and relaxation processes in metals and semiconductors, ${ }^{9-11}$ for example.

${ }^{a)}$ Electronic mail: omatsuda@eng.hokudai.ac.jp
In this article, we describe a theory for laser picosecond acoustics with oblique probe light incidence. The general theory can, in principle, handle arbitrary transparent, semitransparent, or opaque thin multilayer films with optical and elastic anisotropy, taking into account the inhomogeneous modulation of the optical constants therein. However, we focus here on the simple case of a single isotropic opaque material, giving predictions for the changes in reflectance caused by the photoelastic effect and by the surface displacement. We also show how the theory can be applied to allow the experimental separation of these contributions to the reflectance changes.

\section{THEORY}

Consider the situation in which a sample occupies the $z>0$ region and a vacuum occupies the $z<0$ region. We assume that the matter is (macroscopically) invariant under any translation in the $x-y$ plane. The permittivity tensor for all space is given by $\varepsilon_{h}(z)$. Pump light pulses absorbed by the sample generate acoustic pulses in it. In laser picosecond acoustics the diameter of the irradiated region is usually of the order of $10 \mu \mathrm{m}$ or greater, and is much larger than the typical depth of interest in the sample $\leqslant 1 \mu \mathrm{m}$. Thus, the generated acoustic strain pulse can be regarded as a superposition of plane waves propagating in the $z$ direction. The acoustic strain distribution modulates for permittivity tensor spatiotemporally through the photoelastic effect and also creates a time-dependent surface displacement. These perturbations of the permittivity tensor are described as $\varepsilon_{i h}(z, t)$, and result in a change in the (complex) optical reflectance that can be measured with delayed probe light pulses by interferometric methods. ${ }^{3}$ Our object here is to provide an analytical calculation of the reflectance change for arbitrary probe light incident angle and polarization.

To this end, we solve the appropriate electromagnetic wave equation with an inhomogeneous permittivity tensor $\varepsilon_{h}(z)+\varepsilon_{i h}(z, t)$. Because of the relatively low frequency of 


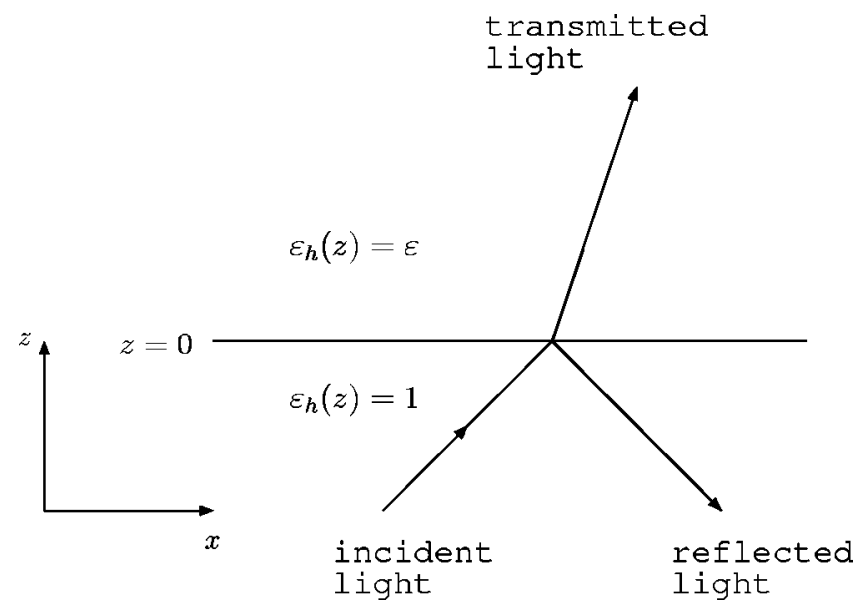

FIG. 1. Incident light comes from $z<0$. All incident, reflected, and transmitted light have common $k_{x}$.

the acoustic perturbation $(<1 \mathrm{THz})$ compared with the frequency of the probe light, we can regard the problem as a quasistatic one. Under this assumption the electromagnetic wave equation for the electric field $\mathbf{E}(\mathbf{x}, t) \exp (-i \omega t)$ is given by

$$
\left[\nabla^{2}-\operatorname{grad} \operatorname{div}+k^{2}\left\{\varepsilon_{h}(z)+\varepsilon_{i h}(z, t)\right\}\right] \mathbf{E}(\mathbf{x}, t)=0,
$$

where $\mathbf{x}$ is the position vector and $k=\omega / c$ is the wave number in vacuum. Let us take the $x$ axis parallel to the plane of incidence, as in Fig. 1. Because the spatial variation in the permittivity tensor takes place only along the $z$ direction, the $x$ component of the wave vector $\left(k_{x}\right)$ is preserved over all space. Therefore, the wave equation is simplified, for $\mathbf{E}(\mathbf{x}, t) \equiv \mathbf{E}(z, t) \exp \left(i k_{x} x\right)$, to

$$
\left[L\left(k_{x}\right)+k^{2}\left\{\varepsilon_{h}(z)+\varepsilon_{i h}(z, t)\right\}\right] \mathbf{E}(z, t)=0,
$$

where

$$
L\left(k_{x}\right) \equiv\left(\begin{array}{ccc}
\frac{\partial^{2}}{\partial z^{2}} & 0 & -i k_{x} \frac{\partial}{\partial z} \\
0 & \frac{\partial^{2}}{\partial z^{2}}-k_{x}^{2} & 0 \\
-i k_{x} \frac{\partial}{\partial z} & 0 & -k_{x}^{2}
\end{array}\right) .
$$

Equation (2) can be solved with the help of a $3 \times 3$ Green's function matrix that satisfies

$$
\left[L\left(k_{x}\right)+k^{2} \varepsilon_{h}(z)\right] G\left(z, z^{\prime}\right)=-\left(\begin{array}{ccc}
1 & 0 & 0 \\
0 & 1 & 0 \\
0 & 0 & 1
\end{array}\right) \delta\left(z-z^{\prime}\right),
$$

and the solution $\mathbf{E}_{0}(z)$ of the homogeneous equation

$$
\left[L\left(k_{x}\right)+k^{2} \varepsilon_{h}(z)\right] \mathbf{E}_{0}(z)=0 .
$$

The resulting solution is expressed as

$$
\begin{aligned}
\mathbf{E}(z, t) & =\mathbf{E}_{0}(z)+\int_{-\infty}^{\infty} k^{2} G\left(z, z^{\prime}\right) \varepsilon_{i h}\left(z^{\prime}, t\right) \mathbf{E}\left(z^{\prime}\right) d z^{\prime} \\
& \simeq \mathbf{E}_{0}(z)+\int_{-\infty}^{\infty} k^{2} G\left(z, z^{\prime}\right) \varepsilon_{i h}\left(z^{\prime}, t\right) \mathbf{E}_{0}\left(z^{\prime}\right) d z^{\prime} .
\end{aligned}
$$

It should be straightforward to extend the above theory to handle arbitrary anisotropic transparent and opaque multilayers. Here, however, we concentrate on the simple case of a single isotropic opaque material (with dielectric constant $\varepsilon)$. The equilibrium dielectric constant $\varepsilon_{h}$ in the absence of any perturbation for all space is given by

$$
\varepsilon_{h}(z)=\left\{\begin{array}{ll}
1 & (z<0) \\
\varepsilon & (z>0)
\end{array} .\right.
$$

The change in the permittivity tensor $\varepsilon_{\text {pe }}$ due to the presence of a longitudinal strain distribution $\eta_{z z}$ is governed by the photoelastic effect:

$$
\varepsilon_{\mathrm{pe}}(z, t)=\left(\begin{array}{ccc}
P_{12} & 0 & 0 \\
0 & P_{12} & 0 \\
0 & 0 & P_{11}
\end{array}\right) \eta_{z z}(z, t),
$$

where $P_{12}$ and $P_{11}$ are photoelastic constants. The surface displacement $u_{0}$ can also be regarded as a change in the permittivity tensor $\varepsilon_{d}$ :

$$
\varepsilon_{d}(z, t)= \begin{cases}\varepsilon-1 & \left(u_{0}(t)<z<0\right) \\ 1-\varepsilon & \left(0<z<u_{0}(t)\right) \\ 0 & \text { elsewhere }\end{cases}
$$

These two contributions give the overall perturbation

$$
\varepsilon_{i h}(z, t)=\varepsilon_{\mathrm{pe}}+\varepsilon_{d} .
$$

Equation (4) for the unperturbed solution has two independent solutions corresponding to (1) $s$ polarization

$$
E_{0}(z)=\left(\begin{array}{l}
0 \\
1 \\
0
\end{array}\right) \times \begin{cases}e^{i k^{\prime} z}+\frac{k^{\prime}-k^{\prime \prime}}{k^{\prime}+k^{\prime \prime}} e^{-i k^{\prime} z} & (z<0), \\
\frac{2 k^{\prime}}{k^{\prime}+k^{\prime \prime}} e^{i k^{\prime \prime} z} & (z>0),\end{cases}
$$

and (2) $p$ polarization

$$
E_{0}(z)=\left\{\begin{array}{l}
\frac{1}{k}\left(\begin{array}{c}
k^{\prime} \\
0 \\
-k_{x}
\end{array}\right) e^{i k^{\prime} z} \\
+\frac{k^{\prime \prime}-\varepsilon k^{\prime}}{k\left(k^{\prime \prime}+\varepsilon k^{\prime}\right)}\left(\begin{array}{c}
k^{\prime} \\
0 \\
k_{x}
\end{array}\right) e^{-i k^{\prime} z} \quad(z<0), \\
\frac{2 k^{\prime}}{k\left(k^{\prime \prime}+\varepsilon k^{\prime}\right)}\left(\begin{array}{c}
k^{\prime \prime} \\
0 \\
-k_{x}
\end{array}\right) e^{i k^{\prime \prime} z} \quad(z>0),
\end{array}\right.
$$

where

$$
k^{\prime}=\sqrt{k^{2}-k_{x}^{2}}, \quad k^{\prime \prime}=\sqrt{\varepsilon k^{2}-k_{x}^{2}} .
$$

The corresponding reflectances are given by 


$$
r_{s}=\frac{k^{\prime}-k^{\prime \prime}}{k^{\prime}+k^{\prime \prime}}, \quad r_{p}=\frac{k^{\prime \prime}-\varepsilon k^{\prime}}{k^{\prime \prime}+\varepsilon k^{\prime}} .
$$

In order to solve the problem, a knowledge of the Green's function matrix is necessary for $z<0$ and $z^{\prime}>0$ in Eq. (5). The nonzero components of the Green's function matrix are given by ${ }^{12}$

$$
\begin{aligned}
& G_{x x}\left(z, z^{\prime}\right)=\frac{i k^{\prime} k^{\prime \prime}}{k^{2}\left(\varepsilon k^{\prime}+k^{\prime \prime}\right)} e^{i\left(k^{\prime \prime} z^{\prime}-k^{\prime} z\right)}, \\
& G_{x z}\left(z, z^{\prime}\right)=\frac{i k^{\prime} k_{x}}{k^{2}\left(\varepsilon k^{\prime}+k^{\prime \prime}\right)} e^{i\left(k^{\prime \prime} z^{\prime}-k^{\prime} z\right),} \\
& G_{y y}\left(z, z^{\prime}\right)=\frac{i}{k^{\prime}+k^{\prime \prime}} e^{i\left(k^{\prime \prime} z^{\prime}-k^{\prime} z\right)}, \\
& G_{z x}\left(z, z^{\prime}\right)=\frac{i k^{\prime \prime} k_{x}}{k^{2}\left(\varepsilon k^{\prime}+k^{\prime \prime}\right)} e^{i\left(k^{\prime \prime} z^{\prime}-k^{\prime} z\right),} \\
& G_{z z}\left(z, z^{\prime}\right)=\frac{i k_{x}^{2}}{k^{2}\left(\varepsilon k^{\prime}+k^{\prime \prime}\right)} e^{i\left(k^{\prime \prime} z^{\prime}-k^{\prime} z\right)} .
\end{aligned}
$$

For small strains and surface displacements, Eq.(5) is transformed into

$$
\begin{aligned}
\mathbf{E}(z, t) \simeq & \mathbf{E}_{0}(z)+\int_{0}^{\infty} k^{2} G\left(z, z^{\prime}\right) \varepsilon_{\mathrm{pe}}\left(z^{\prime}, t\right) \mathbf{E}_{0}\left(z^{\prime}\right) d z^{\prime} \\
& +k^{2} u_{0}(t) G(z,+0)(1-\varepsilon) \mathbf{E}_{0}(-0) .
\end{aligned}
$$

The values for $x= \pm 0$ should be distinguished because the functions have a discontinuity at the interface. The relative reflectance changes for $s$ and $p$ optical polarizations are given by

$$
\begin{aligned}
\frac{\delta r_{s}}{r_{s}}= & 2 i k^{\prime} u_{0}(t)+\frac{2 i k^{\prime} P_{12}}{1-\varepsilon} \int_{0}^{\infty} \eta_{z z}\left(z^{\prime}, t\right) e^{2 i k^{\prime \prime} z^{\prime}} d z^{\prime} \\
\frac{\delta r_{p}}{r_{p}}= & 2 i k^{\prime} u_{0}(t)+\frac{2 i k^{\prime}\left(P_{12} k^{\prime \prime 2}-P_{11} k_{x}^{2}\right)}{k^{\prime \prime 2}-\varepsilon^{2} k^{\prime 2}} \\
& \times \int_{0}^{\infty} \eta_{z z}\left(z^{\prime}, t\right) e^{2 i k^{\prime \prime} z^{\prime}} d z^{\prime}
\end{aligned}
$$

These variations can be directly obtained from experiment.

The above result shows that the surface displacement contribution fortunately appears only in the imaginary part of the relative reflectance changes, and is the same for both $s$ and $p$ polarizations. Thus, the subtraction of Eq. (16) from Eq. (15) allows one to obtain the pure photoelastic contribution to the relative reflectance change, multiplied by a constant complex factor $A$ :

$$
\frac{\delta r_{s}}{r_{s}}-\frac{\delta r_{p}}{r_{p}}=A \int_{0}^{\infty} \eta_{z z}\left(z^{\prime}, t\right) e^{2 i k^{\prime \prime} z^{\prime}} d z^{\prime}=f(t)+i g(t),
$$

where $f(t)$ and $g(t)$ are real functions. Since the real part of Eq. (15) [or Eq. (16)] contains only the photoelastic contribution, it can be expressed by

$$
\operatorname{Re}\left(\frac{\delta r_{s}}{r_{s}}\right)=\operatorname{Re}[B\{f(t)+i g(t)\}]=B_{1} f(t)-B_{2} g(t),
$$

where $B=B_{1}+i B_{2}$ is a complex constant that can be determined by comparing the known functions $f(t), g(t)$, and $\delta r_{s}(t) / r_{s}$. Once the constant $B$ is obtained, the photoelastic contribution to the imaginary part of $\delta r_{s} / r_{s}$ can be calculated, and the displacement contribution to the relative reflectance change is given by

$$
2 k^{\prime} u_{0}(t)=\operatorname{Im}\left(\frac{\delta r_{s}}{r_{s}}\right)-\left\{B_{2} f(t)+B_{1} g(t)\right\} .
$$

This procedure of measuring the response at oblique probe light incidence for $s$ and $p$ polarizations in turn, therefore, allows the separation of the surface-displacement and photoelastic contributions to the relative reflectance change in opaque samples. This remains true for multilayer samples provided that the uppermost layer thickness is larger than the optical penetration depth of the probe light.

\section{SUMMARY}

We have given an analytical treatment of the reflectance variation for light incident at an oblique angle on an opaque isotropic sample whose permittivity tensor is inhomogeneously perturbed, and whose surface is displaced, by a propagating strain wave. It is shown that the surfacedisplacement and photoelastic contributions to the relative reflectance changes can be separated if experimental results are obtained using both $s$ and $p$ polarized probing light.

\section{ACKNOWLEDGMENTS}

The authors thank David Hurley and Takehiro Tachizaki for valuable discussions. This work is partially supported by a Grant-in-Aid for Scientific Research from the Ministry of Education, Science, Sports, and Culture (Japan) and by the Izumi Science and Technology Foundation.

${ }^{1}$ C. Thomsen, H. T. Grahn, H. J. Maris, and J. Tauc, Phys. Rev. B 34, 4129 (1986).

${ }^{2}$ O. B. Wright and K Kawashima, Phys. Rev. Lett. 69, 1668 (1992).

${ }^{3}$ D. H. Hurley and O. B. Wright, Opt. Lett. 24, 1305 (1999).

${ }^{4}$ B. Perrin, B. Bonello, J. C. Jeannet, and E. Romatet, Prog. Nat. Sci. S6, S444 (1996).

${ }^{5}$ O. Matsuda and O. B. Wright, Ultrasonics 40, 753 (2002).

${ }^{6}$ O. Matsuda and O. B. Wright, J. Opt. Soc. Am. B (in press).

${ }^{7}$ D. H. Hurley, O. B. Wright, O. Matsuda, V. E. Gusev, and O. V. Kolosov, Ultrasonics 38, 470 (2000).

${ }^{8}$ O. Matsuda and O. B. Wright, Anal. Sci. 17, S216 (2001).

${ }^{9}$ N. V. Chigarev, D. Y. Paraschuk, X. Y. Pan, and V. E. Gusev, Phys. Rev. B 61, 15837 (2000).

${ }^{10}$ O. B. Wright, Phys. Rev. B 49, 9985 (1994).

${ }^{11}$ O. B. Wright, B. Perrin, O. Matsuda, and V. E. Gusev, Phys. Rev. B 64, R081202 (2001).

${ }^{12}$ A. A. Maradudin and D. L. Mills, Phys. Rev. B 11, 1392 (1975). 